Superalloys 2012: $12^{\text {th }}$ International Symposium on Superalloys

\title{
THE DEVELOPMENT AND VALIDATION OF A NEW THERMODYNAMIC DATABASE FOR NI-BASED ALLOYS
}

\author{
J. Bratberg ${ }^{1}$, H. Mao ${ }^{1,2}$, L. Kjellqvist ${ }^{1}$, A. Engström ${ }^{1}$, P. Mason ${ }^{3}$, Q. Chen ${ }^{1}$ \\ ${ }^{1}$ Thermo-Calc Software AB, Norra Stationsgatan 93 (Plan 5), 11364 Stockholm, Sweden \\ ${ }^{2}$ KTH (Royal Institute of Technology), Brinellvägen 23, 10044 Stockholm, Sweden \\ ${ }^{3}$ Thermo-Calc Software Inc, 4160 Washington Road, Suite 230, McMurray, PA 15317 USA
}

Keywords: Nickel, Modeling, CALPHAD, Phase Equilibria, Diffusion, Precipitation

\begin{abstract}
Thermodynamic databases developed using the CALPHAD method has been successfully applied to the modeling and simulation of $\mathrm{Ni}$ based superalloys for more than fifteen years. Such databases when combined with suitable software, such as Thermo-Calc, can be used for accelerating alloy design as well as improving understanding of existing alloys in terms of their processing and in-service behavior. Additionally, such databases are also essential to the modeling of microstructural evolution using methods such as phase field or mean field approaches.
\end{abstract}

A new thermodynamic database, TCNI6, has been developed for Ni-based superalloys based on the critical evaluation, using the CALPHAD method, of all the constituent binary systems across their full range of composition and many of the ternary systems containing $\mathrm{Ni}$, as well as a number of other key ternary systems. The database has also been validated where possible against higher order systems as well.

Together with the new mobility database, MOBNI3, which contains descriptions for the atomic mobility for respectively the $\gamma$ (A1), $\gamma^{\prime}\left(\mathrm{L1}_{2}\right), \alpha$ (A2), $\beta$ (B2) and liquid phases, TCNI6 can be used with software such as DICTRA to predict different kinetic aspects taking all relevant phases into account, e.g. the simulation of interdiffusion across Ni-based coatings.

A new user friendly computational tool, TC-PRISMA, which simulates the kinetics of precipitation processes, is demonstrated utilizing these new databases and combined with interface property data. Engineering applications of precipitation modelling are validated against experimental data.

\section{Introduction}

Ni-based superalloys are typically connected to applications in aggressive environments under demanding conditions and require properties such as high-temperature strength, good resistance against creep, fatigue, resistance to oxidation, and hot corrosion. In order for these requirements to be met, Ni-based superalloys usually contain at least 10 alloying elements, with each one being added for a specific purpose. Due to this complexity in chemistry, it takes traditionally a long time to optimize properties of existing alloys and to develop completely new alloys. Thanks to computational thermodynamics or the so-called CALPHAD technique $[1,2]$, it is now possible to perform realistic calculations of phase diagrams and phase transformations in multi-component systems. CALPHAD type modeling has proven very useful in the design of Ni-based superalloys by providing an accessible understanding of the influence from chemistry and processing conditions on the microstructures consisting of multiple phases. The key feature of this technique is to model the
Gibbs energy of each individual phase in a way that permits prediction of thermodynamic properties of multicomponent systems from those of binary and ternary subsystems. Its huge success relies on the development of consistent databases where each phase is described separately using models based on physical principles and model parameters assessed from experimental and first principles calculation data. The quality of the predictions based on calculations and simulations depends highly on the quality of the thermodynamic and kinetic databases. The modeling and simulation studies by using flexible software and accurate databases can significantly reduce the cost and lead time for alloy development and process improvement.

This paper describes the development and validation of a new thermodynamic database, TCNI6 [3], for Ni-based alloy systems. It contains 25 chemical elements and 343 phases. This database has been developed using the CALPHAD approach with industry input and support. It is based on the critical evaluation of binary, ternary and in some cases higher order systems which enables predictions for multicomponent systems and industrially important alloys. The database has also been validated where possible against higher order systems.

Using the Thermo-Calc software [4, 5], TCNI6 allows us to calculate the thermodynamic equilibrium of complex alloys during the solidification as well as at different key temperatures for the industrial heat treatments or in-service ageing. It enables us to better understand the influence of different elements on the stability of phases, their fraction and their composition. Through such calculations it is possible to optimize compositions and dissolution and precipitation heat treatment temperature for new or existing alloys by performing a significantly reduced number of time-consuming and costly experiments.

Together with the mobility database, MOBNI3 [6], kinetic reactions can also be simulated with the use of DICTRA [7] or TC-PRISMA [8]. DICTRA is a computer program for simulating diffusion controlled phase transformations in multicomponent alloy systems such as homogenization/aging of alloys, dissolution/coarsening of precipitates. Since all necessary volume data (including molar volume and thermal expansion) for various alloy phases have been incorporated, it allows for the calculation of volume fraction of phases, as well as density, thermal expansion and lattice parameters, e.g. misfits between $\gamma$ and $\gamma$ '. Molar volumes are important for precipitation modeling using TC-PRISMA (together with driving forces and mobilities). TCPRISMA is a computational tool for the simulation of multiparticle precipitation reactions in multicomponent alloys. 


\section{Thermodynamic and kinetic models}

TCNI6 is a self-consistent thermodynamic database developed using the CALPHAD approach for the application of Ni-based superalloys. The heart of the CALPHAD approach is the coupling of phase diagrams and thermochemistry through self-consistent numerical models allowing easy and trustful multicomponent extrapolations. With the CALPHAD technique an internally consistent thermodynamic description can be obtained to describe both phase equilibria and the thermodynamics of a system. Each phase of the system under consideration is described using a thermodynamic model which describes the Gibbs energy as a function of temperature, pressure and composion. The model parameters are estimated by the weighted nonlinear least-square optimization of phase diagram and thermochemical data. Thermodynamic modeling is an essential factor for the success of an optimization and for the predictive power of a self-consistent dataset. In general [9], the molar Gibbs energy $\left(G_{m}\right)$ of a phase at $1 \mathrm{~atm}$ pressure is expressed by

$$
G_{m}={ }^{s r f} G_{m}+{ }^{p h y s} G_{m}-T \cdot{ }^{c o n f} S_{m}+{ }^{E} G_{m}
$$

where the superscript "srf" stands for "surface of reference" and the value of ${ }^{s r f} G_{m}$ is the weighted average of Gibbs energy of the components of the phase relative to their reference state. The term ${ }^{p h y s} G_{m}$ represents the contribution to the Gibbs energy from some particular physical phenomenon such as the magnetic contribution. The configurational entropy of the phase is denoted as ${ }^{c o n f} S_{m}$. The last term ${ }^{E} G_{m}$ in Eq. (1) stands for the excess Gibbs energy which describes the remaining part of the real Gibbs energy of the phase after the first three terms have been subtracted. The temperature dependence of the Gibbs energy of a stoichiometric phase or an end-member of a solution phase $\left({ }^{\circ} G_{m}\right)$ is described by a polynomial like

$$
{ }^{o} G_{m}-\Sigma b_{i} H_{i}^{S E R}=A+B T+C T \ln (T)+\ldots
$$

where $b_{i}$ is the stoichiometric factor of the element $i$ in the phase and $\Sigma b_{i} H_{i}^{S E R}$ represents the sum of the enthalpies of the elements in their reference state, usually the stable state at $298.15 \mathrm{~K}$ and 1 atmosphere, denoted SER. For solution phases, the Compound Energy Formalism (CEF) [10] is implemented with a multisublattice model where a constituent (denoted $i$ ) is a certain species in a certain sublattice, and a constituent array (denoted I) specifies one or more constituents on each sublattice (denoted s). The constituent arrays can be of different order. The zeroth-order $\left(I_{0}\right)$ has just one constituent on each sublattice and describes an end-member of the solution phase. The surface of reference ${ }^{\mathrm{srf}} \mathrm{G}_{\mathrm{m}}$ for the Gibbs energy of a phase by CEF is expressed by

$$
{ }^{s r f} G_{m}=\Sigma\left(G_{m}\left(I_{0}\right) \cdot \Pi y_{I_{0}}\right)
$$

where $\Pi y_{I_{0}}$ is the product of the site fractions, i.e the mole fractions of the constituent $I_{0}$ on specific sublattices. The summation covers all the end-members. ${ }^{\circ} G_{m}\left(I_{0}\right)$ is the Gibbs energy of one mole of formula units of the compound representing the end-member. The quantity $\Sigma\left(\Pi y_{I_{0}}\right)$ is unity and ${ }^{s r f} G_{m}$ thus represents a weighted average over all the end-members. The configurational entropy ${ }^{c o n f} S_{m}$ is expressed by

$$
{ }^{c o n f} S_{m}=-R \sum_{s=1}^{n} a_{s} \sum_{i=1}^{n_{s}} y_{i}^{(s)} \ln \left(y_{i}^{(s)}\right)
$$

where the factor $a_{s}$ is the number of sites on each sublattices, $y_{i}^{(s)}$ denotes the site fraction of $i$ on $s$. The first sum covers all sublattices and the second covers all constituents on each sublattice. Such an expression of the configurational entropy is actually based on the assumption of random mixing within each sublattice. The excess Gibbs energy ${ }^{E} G_{m}$ is expressed by

$$
{ }^{E} G_{m}=\Sigma\left(L_{I_{1}} \cdot \Pi y_{I_{l}}\right)+\Sigma\left(L_{I_{2}} \cdot \Pi y_{I_{2}}\right)+\ldots \ldots
$$

which contains all possible interactions defined by the constituent array of different orders. The first-order constituent array $\left(I_{I}\right)$ has a second constituent in one of the sublattices. In other words, there is a binary excess contribution from $I_{l}$. The second-order array $\left(I_{2}\right)$ includes a ternary parameter and/or a so-called reciprocal parameter. The ternary parameter implies three interacting constituents on one specific sublattice when all the other sublattices are occupied only by one single constituent respectively. The reciprocal parameter implies two interacting constituents on two different sublattices while all others are occupied by only one constituent. The higher order arrays are not used. The $L$ parameters could depend on temperature and composition. The binary interaction parameters are usually expanded by the Redlich-Kister polynomial in the terms of site fractions.

$$
L_{i j}=\sum_{v=0}^{k}\left(y_{i}-y_{j}\right)^{v} \cdot{ }^{v} L_{i j}
$$

where the ${ }^{v} L_{i j}$ parameter can normally be linearly temperature dependent.

Within the CEF framework, the unique ionic two-sublattice model $[11,12]$ was used for both the metallic and ionic liquid. The model assumes that cations mix on one sublattice and anions on the other. In order to extend the model description to a liquid with only cations (i.e. a metallic liquid) hypothetical vacancies were introduced. For non-metallic liquids neutral species were also allowed in the anion sublattice. The model can be written generally as

$$
\left(C_{i}{ }^{v}\right)_{P}\left(A_{j}{ }^{v}, V a^{-Q}, B_{k}{ }^{0}\right)_{Q}
$$

where each pair of parentheses surround a sublattice. $C$ represents cations, $A$ anions, $V a$ vacancies and $B$ neutrals. The index $i$ denotes a specific constituent and $v_{i}$ its charge. The electroneutrality is maintained by varying the site numbers $P$ and $Q$ as the average charge on the other sublattice.

$$
\begin{aligned}
& P=\boldsymbol{\Sigma}\left(-v_{j}\right) y_{A_{j}}+Q y_{V a} \\
& Q=\Sigma\left(v_{i}\right) y_{C_{i}}
\end{aligned}
$$

where y denotes the site fraction.

Some intermediate phases represent ordered forms of the BCC, FCC and HCP lattices. These should be modeled with an order/disorder model even if the disordered phase is not stable in the system. A critical fact is that the ordered phases must disorder 
correctly when the ordering is no longer stable. This is achieved by partitioning the Gibbs energy in two parts.

$$
\begin{aligned}
& G_{m}=G_{m}{ }^{d i s}(x)+\Delta{G_{m}}^{\text {ord }}(y) \\
& \Delta{G_{m}}^{\text {ord }}=G_{m}{ }^{s l}(y)-G_{m}{ }^{s l}(y=x)
\end{aligned}
$$

where $x$ and $y$ are mole fraction and site fraction, respectively. $\Delta G_{m}{ }^{\text {ord }}$ equals to zero when the phase is disordered.

The A2/B2 ordering in BCC can be modeled with two sublattices, e.g. in the $\mathrm{Al}-\mathrm{Ni}$ binary system as $(\mathrm{Al}, \mathrm{Ni})_{0.5}(\mathrm{Al}, \mathrm{Ni})_{0.5}$. B2 ordering requires $\mathrm{G}(\mathrm{B} 2, \mathrm{Ni}: \mathrm{Al})=\mathrm{G}(\mathrm{B} 2, \mathrm{Al}: \mathrm{Ni})$ as the two sublattices are identical. All interaction parameters on both sublattices must also be equal, i.e. $\mathrm{L}(\mathrm{B} 2, \mathrm{Al}, \mathrm{Ni}: \mathrm{Al})=\mathrm{L}(\mathrm{B} 2, \mathrm{Al}: \mathrm{Al}, \mathrm{Ni})$. There are often substitutional vacancies in the $\mathrm{B} 2$ phase, yielding the $\mathrm{B} 2$ model as $(\mathrm{Al}, \mathrm{Ni}, \mathrm{Va})_{0.5}(\mathrm{Al}, \mathrm{Ni}, \mathrm{Va})_{0.5}$. Thus in the disordered $\mathrm{BCC}$ phase one then has to introduce "thermal vacancies" in the substitutional lattice. In addition, the introduction of a third sublattice for the interstitial sites gives the following thermodynamic model e.g. of the $\mathrm{Al}-\mathrm{Ni}-\mathrm{C}$ system as $(\mathrm{Al}, \mathrm{Ni}, \mathrm{Va})_{0.5}(\mathrm{Al}, \mathrm{Ni}, \mathrm{Va})_{0.5}(\mathrm{Va}, \mathrm{C})_{3}$

The $\mathrm{A} 1 / \mathrm{L} 1_{2}$ ordering in $\mathrm{FCC}$ can also be modeled with two sublattices (or three with interstitials) as e.g. $(\mathrm{Al}, \mathrm{Ni})_{0.75}(\mathrm{Al}, \mathrm{Ni})_{0.25}$ $(\mathrm{Va}, \mathrm{C})_{1}$. This model has many complicated relations between the parameters because there are nearest neighbor bonds both between the sublattices and within the sublattice with 0.75 sites. More details of the order-disorder modeling can be found in Dupin and Sundman [13].

The topologically closed-packed (TCP) phases (such as $\sigma, \mu, \mathrm{P}, \mathrm{R}$ and Laves phase) are described using combined CEF as presented in Hallstedt et al. [14] resulting from the group discussion within the CALPHAD research community. For instance the sigma phase $(\sigma)$ often modeled in the past as $(\mathrm{A}, \mathrm{B})_{10}(\mathrm{~A})_{4}(\mathrm{~A}, \mathrm{~B})_{16}$ ignoring the very low degree of order of this phase in some systems, like $\mathrm{Cr}-\mathrm{Fe}$ for instance, is described with all the elements in all sub-lattices i.e. $(\mathrm{Co}, \mathrm{Cr}, \mathrm{Re})_{10}(\mathrm{Co}, \mathrm{Cr}, \mathrm{Re})_{4}(\mathrm{Co}, \mathrm{Cr}, \mathrm{Re})_{16}$ in the $\mathrm{Co}-\mathrm{Cr}-\mathrm{Re}$ ternary system. The molar Gibbs energy is given by

$G_{m}^{\sigma}=G_{m}^{\mathrm{dis}}\left(x_{i}\right)+\Delta G_{m}^{\mathrm{conf}}\left(y_{i}^{s}\right)$,

$G_{m}^{\mathrm{dis}}\left(x_{i}\right)=\sum_{i} x_{i}{ }^{\circ} G_{i}^{\sigma}+\sum_{i} \sum_{j>i} \sum_{v} x_{i} x_{j}\left(x_{i}-x_{j}\right)^{v} L_{i, j}^{v, \sigma}$

where

$$
\begin{aligned}
\Delta G_{m}^{\mathrm{conf}}\left(y_{i}^{s}\right)= & \sum_{i} \sum_{j} \sum_{k} y_{i}^{(1)} y_{j}^{(2)} y_{k}^{(3)} \Delta G_{i: j: k}^{\sigma} \\
& +a^{(s)} R T \sum_{s} \sum_{i} y_{i}^{(s)} \ln y_{i}^{(s)},
\end{aligned}
$$

$x_{i}$ being the overall composition of the $\sigma$ phase,

$y_{i}^{(s)}$, the fraction of the site $s$ occupied by the element $i$,

$a^{(s)}$, the fraction of the total number of sites corresponding to site $s\left(a^{(1)}=10 / 30, a^{(2)}=4 / 30, a^{(3)}=16 / 30\right)$,

${ }^{\circ} G_{i}^{\sigma}$, the molar Gibbs energy of the pure element $i$ in the hypothetical $\sigma$ state,

$L_{i, j}^{\nu, \sigma}$, binary interaction parameters,

$\Delta G_{i: j: k}^{\sigma}$, the molar Gibbs energy of formation of the stoichiometric compound $i_{a^{(1)}} j_{a^{(2)}} k_{a^{(3)}}$ referred to the elements in the hypothetical $\sigma$ state.
The model used to describe the molar volume at ambient pressure is derived from the definition of the coefficient of linear thermal expansion (CLE) $\alpha$ as

$$
\mathrm{V}_{\mathrm{m}}(\mathrm{T})=\mathrm{V}_{0} \exp \left(\int_{T 0}^{T} 3 \alpha \mathrm{dT}\right)+\Delta \mathrm{V}_{\mathrm{m}}{ }^{\mathrm{magn}}(\mathrm{T})
$$

where $\mathrm{V}_{0}$ is the molar volume at the reference temperature, T0. $\Delta \mathrm{V}_{\mathrm{m}}{ }^{\text {magn }}(\mathrm{T})$ is the magnetic contribution to the molar volume. Details about this model and its implementation in Thermo-Calc can be found in Lu et al. [15].

The mobility database (MOBNI3) contains descriptions for the atomic mobility. The mobility e.g. for element $B\left(M_{B}\right)$ is a kinetic parameter that gives the rate of exchange if there is a vacant atom site adjacent to $\mathrm{a}$ atom. It is given by

$$
M_{B}=M_{B}^{0} \exp \left(\frac{-Q_{B}}{R T}\right) \frac{1}{R T}
$$

The pre-exponential factor $\mathrm{M}_{\mathrm{B}}{ }^{0}$ referred to as the frequency factor is related to the number of jump attempts (or jump frequency) for an atom. The exponential factor $\left(-\mathrm{Q}_{\mathrm{B}}\right)$ is used for the activation energy which is related to the probability of a successful jump and may be evaluated from the theory of thermal fluctuations. When treating the composition dependency of the mobility it has been found superior to expand the logarithm of the mobility rather than the value itself, i.e.

$$
R T \ln \left[R T M_{B}\right]=R T \ln M_{B}^{0}-Q_{B}
$$

The composition dependency is represented with a linear combination of the values at each endpoint of the composition space, and a Redlich-Kister expansion, i.e.

$$
\Phi_{\mathrm{B}}=\sum_{i} x_{i} \Phi_{B}^{i}+\sum_{i} \sum_{j>i} x_{i} x_{j}\left[\sum_{r=0}^{m}{ }^{r} \Phi_{B}^{i, j}\left(x_{i}-x_{j}\right)^{r}\right]
$$

Where $\Phi_{\mathrm{B}}$ represents $\mathrm{RT} \ln \mathrm{M}_{\mathrm{B}}{ }^{0}$ or $\mathrm{Q}_{\mathrm{B}}$.

To take into account the influence from chemical-ordering on the mobillities in the $\beta$ (B2) and $\gamma^{\prime}\left(\mathrm{L1}_{2}\right)$ phases, the phenomenological model suggested by Helander and Ågren [16] is used. According to this model $\mathrm{Q}_{\mathrm{B}}$ is given by

$$
Q_{B}=Q_{B}^{d i s}+\Delta Q_{B}^{\text {ord }}
$$

The last term counts the summation of contributions to the activation energy from chemical ordering between different $i$ and $j$ atoms, i.e.

$$
\Delta Q_{B}^{\text {ord }}=\sum_{i} \sum_{i \neq j} \Delta Q_{B i j}^{\text {order }}\left[y_{i}^{\alpha} y_{j}^{\beta}-x_{i} x_{j}\right]
$$

\section{Development of TCNI6 and MOBNI3}

TCNI6 contains 25 elements namely $\mathrm{Al}, \mathrm{Ar}, \mathrm{B}, \mathrm{C}, \mathrm{Co}, \mathrm{Cr}, \mathrm{Fe}, \mathrm{H}$, Hf, Mo, N, Nb, Ni, O, Pd, Pt, Re, Ru, Si, Ta, Ti, V, W, Y, Zr. It is based on the critical evaluation of all the constituent binary systems across their full range of composition and many of the ternary systems containing $\mathrm{Ni}$, as well as a number of other key 
ternary systems. The database includes 343 solution and intermetallic phases. The ordered and disordered bcc (A2/ $\alpha$ and $\mathrm{B} 2 / \beta)$ and $\mathrm{fcc}\left(\mathrm{A} 1 / \gamma\right.$ and $\left.\mathrm{L} 12 / \gamma^{\prime}\right)$ phases are modeled with a two sub-lattice model using a single Gibbs energy curve, and this type of description is of particular importance to be able to predict second order transformations e.g. between A2 and B2. The TCP phases are modeled using complex and crystallographically based models, which gives the ability to correctly predict site-fractions and the wide range of stability of these phases. Oxygen has been implemented in an ambitious way using the CEF for the solid solution phases. For example, in the case of the $\mathrm{Cr}-\mathrm{Fe}-\mathrm{Ni}-\mathrm{O}$ system the spinel phase is modeled as $\left(\mathrm{Cr}^{+2}, \mathrm{Cr}^{+3}, \mathrm{Fe}^{+2}, \mathrm{Fe}^{+3}, \mathrm{Ni}^{+2}\right)_{1}$ $\left(\mathrm{Cr}^{+3}, \mathrm{Fe}^{+2}, \mathrm{Fe}^{+3}, \mathrm{Ni}^{+2}, \mathrm{Va}\right)_{2}\left(\mathrm{Cr}^{+2}, \mathrm{Fe}^{+2}, \mathrm{Va}\right)_{2}\left(\mathrm{O}^{-2}\right)_{4}$, the halite phase as $\left(\mathrm{Cr}^{+3}, \mathrm{Fe}^{+2}, \mathrm{Fe}^{+3}, \mathrm{Ni}^{+2}, \mathrm{Ni}^{+3}, \mathrm{Va}\right)_{1} \quad\left(\mathrm{O}^{-2}\right)_{1}$, and corundum as $\left(\mathrm{Cr}^{+2}, \mathrm{Cr}^{+3}, \mathrm{Fe}^{+2}, \mathrm{Fe}^{+3}\right)_{2}\left(\mathrm{Cr}^{+3}, \mathrm{Fe}^{+3}, \mathrm{Ni}^{+2}, \mathrm{Va}\right)_{1}\left(\mathrm{O}^{-2}\right)_{3}$. Details of the modeling on ionic systems can be found in Hillert et al. [17] and for the Cr-Fe-Ni-O case in Kjellqvist et al. [18]. Molar volume data and thermal expansivities are critically assessed for most phases of importance to Ni-based superalloys including $\gamma$ (A1), $\gamma$ ' (L12), $\alpha$ (A2), $\beta$ (B2), liquid, $\gamma$ " (DO22), $\eta$ (DO24), $\delta$ (DOa), TCP-phases (such as $\sigma, \mu, \mathrm{P}, \mathrm{R}$ and Laves phase), carbides (such as $\mathrm{MC}, \mathrm{M}_{3} \mathrm{C}_{2}, \mathrm{M}_{7} \mathrm{C}_{3}, \mathrm{M}_{23} \mathrm{C}_{6}$ and $\mathrm{M}_{6} \mathrm{C}$ ) and borides (such as $\mathrm{M}_{3} \mathrm{~B}_{2}$, $\mathrm{M}_{5} \mathrm{~B}_{3}$ ). All intermetallic phases are assigned a molar volume, also those where no experimental information is found, by using a linear combination of parameters.

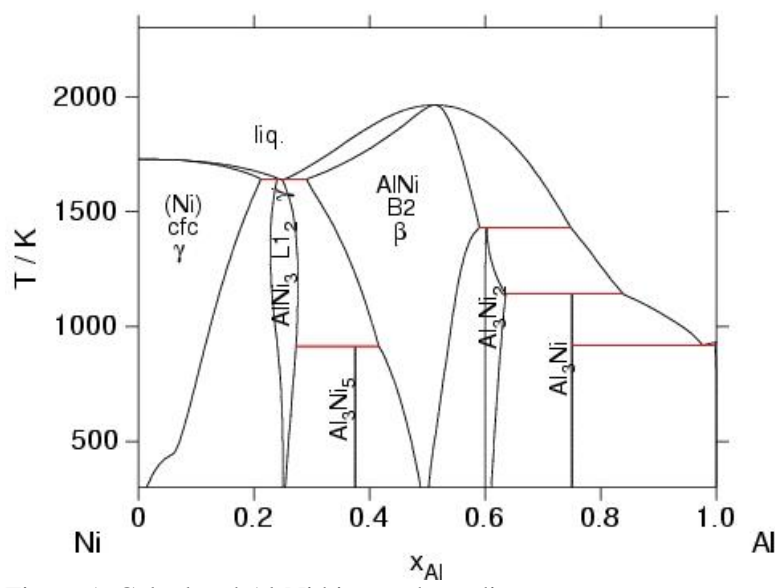

Figure 1. Calculated Al-Ni binary phase diagram.

Combined with Thermo-Calc, TCNI6 can be used to predict thermodynamic properties and phase diagrams for multicomponent systems. The predictive capacity of a thermodynamic database depends on the quality of the thermodynamic modeling and assessments of low-order systems.

Using the above mentioned order-disorder modeling, the Al-Ni phase diagram including both the ordered B2 and L12 phases has been calculated and is illustrated in Figure 1. The second order structural transition for BCC is evident in Figure 2, showing the calculated Al-Fe binary phase diagram.

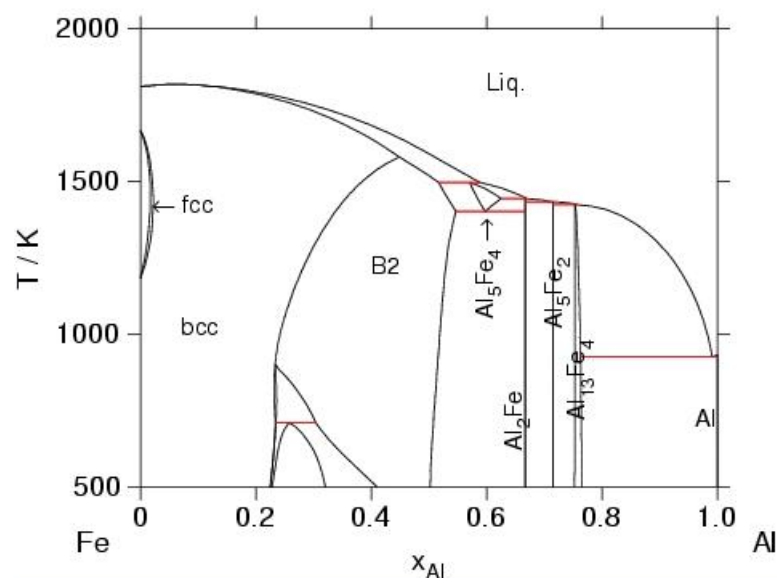

Figure 2. Calculated Al-Fe binary phase diagram.

With respect to TCP phases, Figure 3 demonstrates a smooth description in the $\mathrm{Co}-\mathrm{Cr}-\mathrm{Re}$ isothermal section between $\mathrm{Co}-\mathrm{Cr}$ and $\mathrm{Cr}-\mathrm{Re}$ sigma phases which have different compositions.

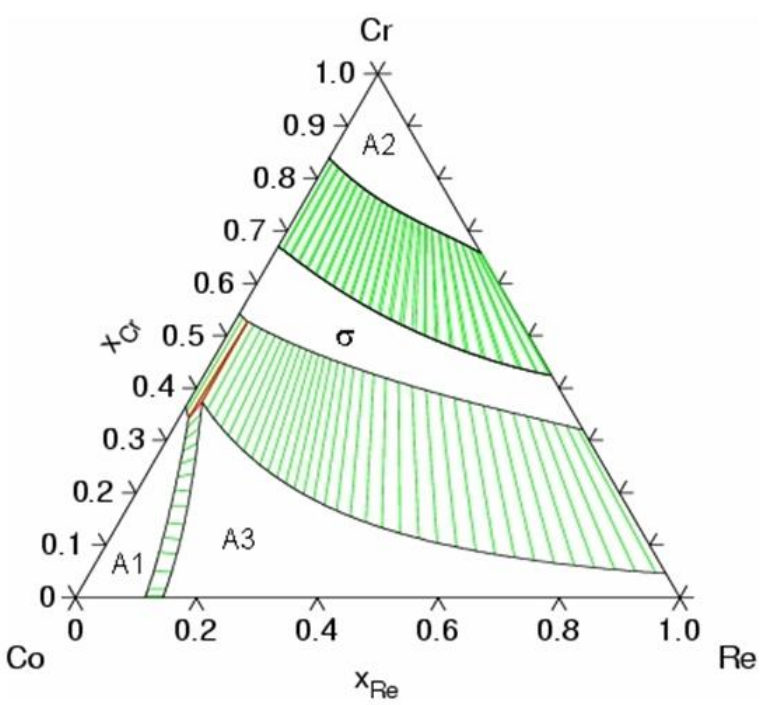

Figure 3. Isothermal section of the Co-Cr-Re system at $1273 \mathrm{~K}$.

TCNI6 also reflects an updated thermodynamic database by the inclusion of new assessments or reassessments of some low-order systems of the Ni-based alloys. For instance, the Al-Co-Ni-W quaternary system has been revised taking into account the recent experimental data in this quaternary with high content of $\mathrm{Co}$, where the Al-Co-W ternary is a new evaluation. Figure 4 compares the calculated and experimental isoplethal sections at equal-mole amounts of $\mathrm{Al}$ and $\mathrm{W}$ of the $\mathrm{Al}-\mathrm{Co}-\mathrm{W}$ system. Figure 5 shows the isothermal section at $1173 \mathrm{~K}$ and with 30 at\% Ni for the quaternary Al-Co-Ni-W system. Symbols represent experimental data of Dmitrieva [19] in Figure 4, and Shinagawa et al. [20] in Figure 5. Solid curves are calculated using the present TCNI6, dashed ones by an earlier description present from TCNI4, and dotted ones by the recent description of Cui et al [21]. It can be seen in both figures that significant improvements are achieved with the present description. 


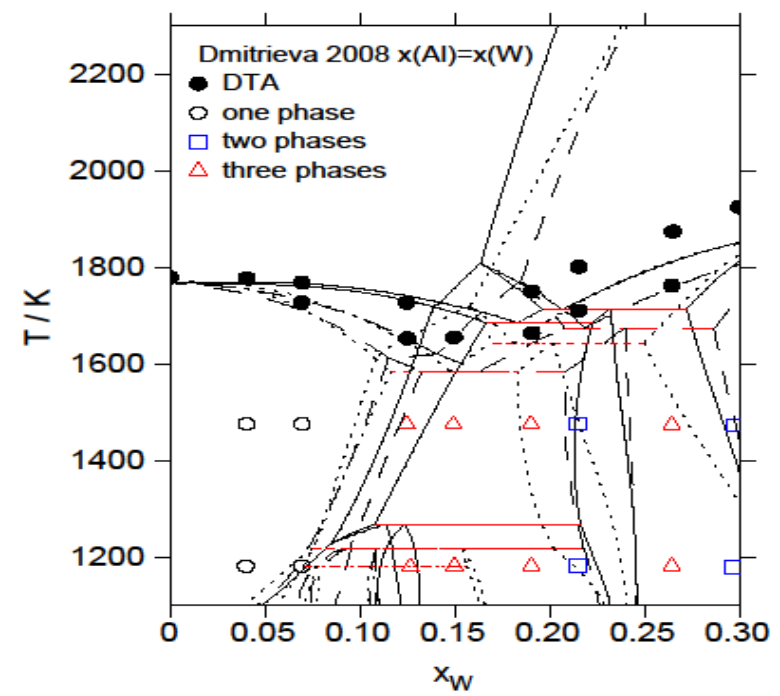

Figure 4. Monovariant lines of the liquidus and solidus surface at equal-mole of $\mathrm{Al}$ and $\mathrm{W}$ of the $\mathrm{Al}-\mathrm{Co}-\mathrm{W}$ system.

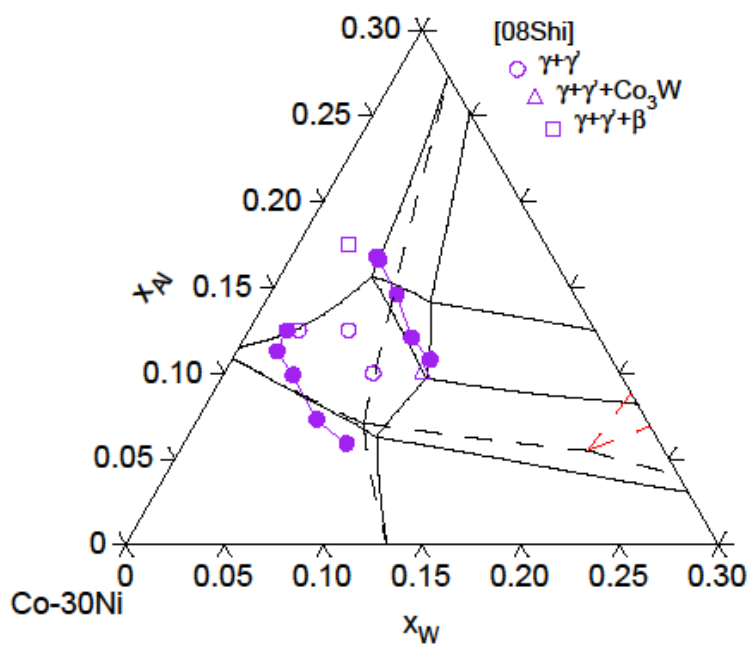

Figure 5. Isothermal section at $1173 \mathrm{~K}$ and 30 at.\% $\mathrm{Ni}$ of the quaternary Al-Co-Ni-W system.

Using the thermodynamic model for the ordered phases that do not disorder, such as the sigma phase, then the model-predicted site fraction of the sigma phase of the Pd-Ta system compares well with the experimental data from Joubert [22], see Figure 6. The agreement is impressive thanks to the more physically reasonable thermodynamic model that is used here. The first sublattice describes the Wyckstoff position $2 \mathrm{a}$ and $8 \mathrm{i} 2$, the second sub-lattice describes the Wyckstoff position $4 \mathrm{f}$, and the third sublattice describes the Wyckstoff position $8 \mathrm{i} 1$ and $8 \mathrm{j}$. Using the combined CEF with 3 sub-lattices for the sigma phase allows keeping close relation to crystallography with reasonable number of assessed parameters. When ab initio data are available, it can be directly taken into account in the configurational contribution.

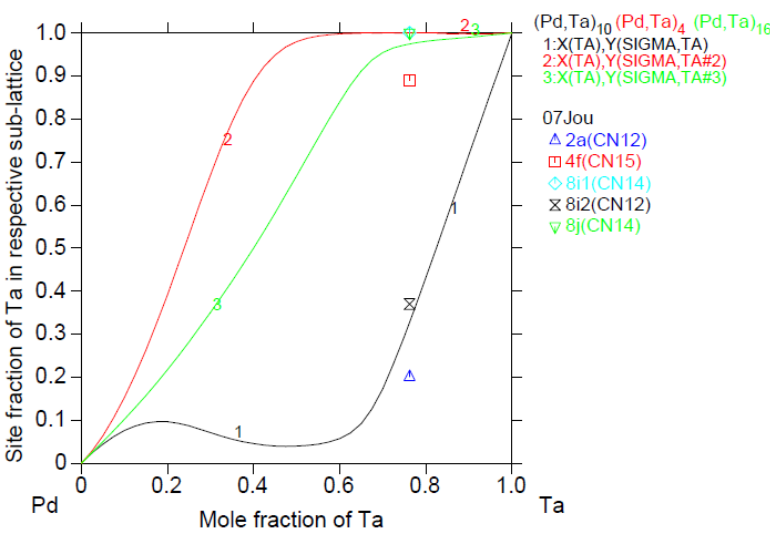

Figure 6. Predicted site occupations of Ta in the different sublattices of the sigma phase in the Pd-Ta system at $1923 \mathrm{~K}$ compared to experimental data from Joubert [22].

A new mobility database (MOBNI3) has also been developed that is compatible with TCNI6. Here compatibility means that the descriptions of elements and phases are identical in both databases and the mobility parameters have been optimized using TCNI6, i.e. the thermodynamic factors required during the optimization of mobility parameters against experimental inter- or intrinsicdiffusion data were calculated based on TCNI6 thermodynamics. MOBNI3 contains descriptions for the atomic mobility for respectively $\gamma(\mathrm{A} 1), \gamma^{\prime}\left(\mathrm{L1}_{2}\right), \alpha(\mathrm{A} 2), \beta$ (B2) and liquid phase.

Figure 7 and 8 demonstrate example of optimization result for $\gamma$ (A1) and $\beta$ (B2), respectively. In Figure 7 the calculated interdiffusion coefficient in the $\gamma$-phase (A1) for the Co-Pt system at various temperatures is compared with the experimental data taken from Iijima et al [23].

Whereas Figure 8 shows a comparison between calculated and experimentally determined $\mathrm{Pt}$ tracer diffusion coefficients in $\mathrm{NiAl}$ compounds with compositions of $43.7-51.9$ at.\% $\mathrm{Al}$ in a temperature range 1373 to $1673 \mathrm{~K}$.

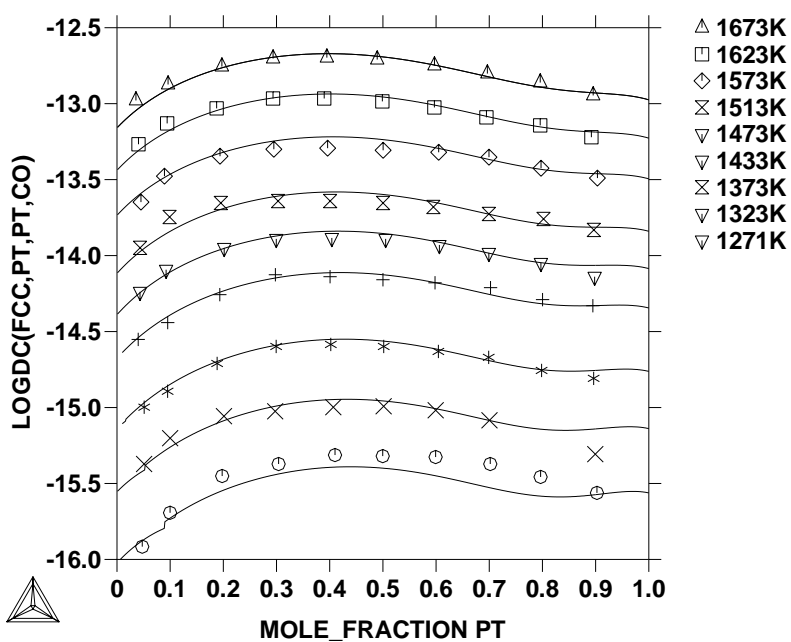

Figure 7. The interdiffusion coefficient in FCC_A1 of the Co-Pt binary system. Symbols are experimental data at various temperatures taken from Iijima et al [23]. 


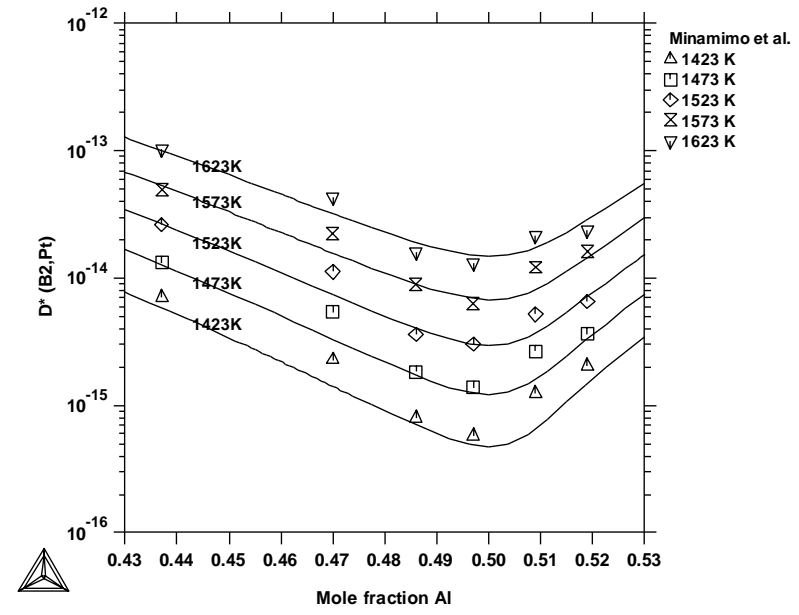

Figure 8. Comparison of the calculated and experimental (Minamino et al. [24]) concentration dependence of tracer diffusion coefficients of $\mathrm{Pt}$ in the $\mathrm{NiAl}$ alloys.

\section{Examples of calculation and simulation}

Based on the assessments of low-order systems, the database is validated where possible against higher order systems, such as data published for industrial alloys. Figure 9 predicts the mole fraction of thermodynamically stable phases between $600{ }^{\circ} \mathrm{C}$ and $1200{ }^{\circ} \mathrm{C}$ for the TMW-4 superalloy with composition $\mathrm{Ni}-15 \mathrm{Cr}$ $26 \mathrm{Co}-2.8 \mathrm{Mo}-1.1 \mathrm{~W}-1.9 \mathrm{Al}-6 \mathrm{Ti}$ (wt. \%). The prediction is in excellent agreement with the experimental data e.g. the eta phase stable between $1030{ }^{\circ} \mathrm{C}$ to $1170{ }^{\circ} \mathrm{C}$ as reported by Cui [25]

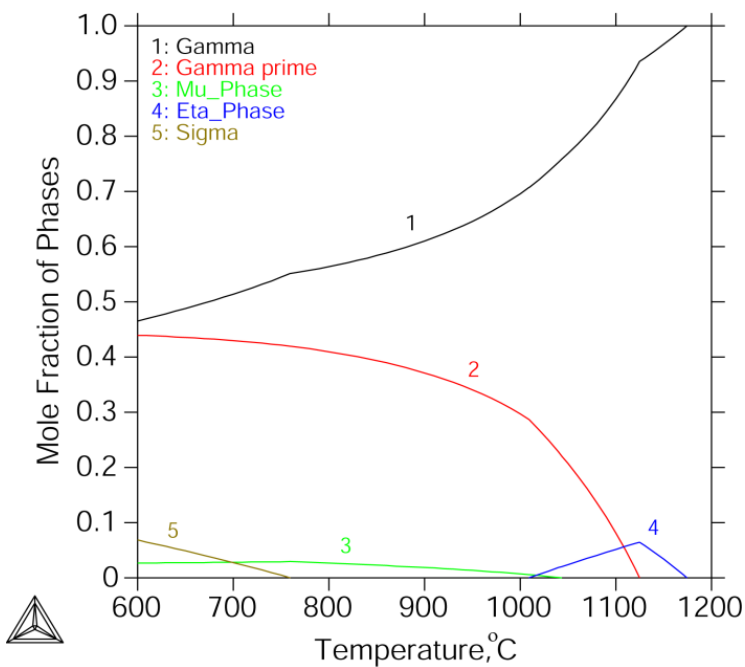

Figure 9. Predicted amount of phases (NI3TI_D024 is eta phase) at varying temperatures for a TMW-4 alloy with $\mathrm{Ni}-15 \mathrm{Cr}-26 \mathrm{Co}-$ 2.8Mo-1.1W-1.9Al-6Ti (wt. \%).

Phase transformations usually occur together with volume changes. Using TCNI6, one can calculate volumetric properties e.g. molar volumes, densities, lattice parameters (for phases with cubic structures), thermal expansion etc. of a given alloy system. Figure 10 shows the liquid densities varying with temperature for the Ni-Cr-Al-Mo alloy at various Mo content (in mass \%). The molar ratio of $\mathrm{Ni}: \mathrm{Cr}: \mathrm{Al}$ (73:14:13) is close to the average value for commercial superalloys INCO713, TMS75, CM247LC and CMSX-4. The symbols represent measured densities of liquid NiCr-Al-Mo alloys by Fang et al. [26]. The agreement is satisfactory.

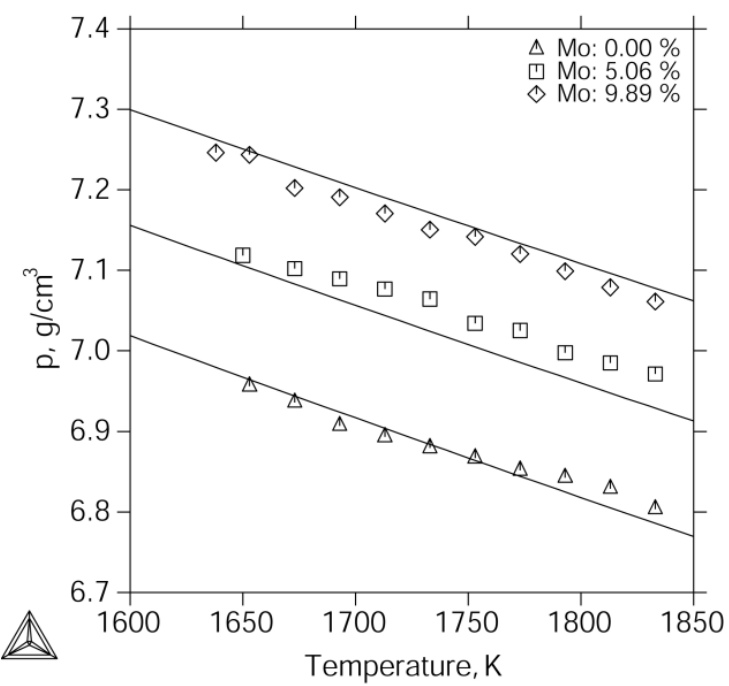

Figure 10. Predicted densities of liquid Ni-Cr-Al-Mo alloys where the molar ratio of Ni:Cr:Al is 73:14:13. Symbols are the experimental values from Fang et al. [26].

Using high temperature X-ray diffraction Nathal et al. [27] determined the $\gamma / \gamma$ ' lattice mismatch of Ni-base superalloys from room temperature up to $1000{ }^{\circ} \mathrm{C}$. The measured temperature dependence of lattice parameters in commercial NASAIR 100 superalloy is compared in Figure 11 with the predictions according to TCNI6.

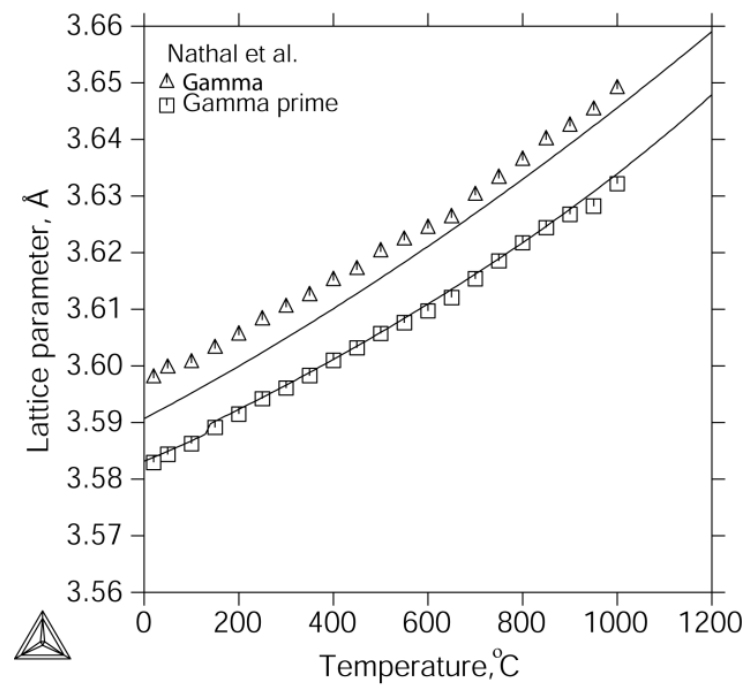

Figure 11. Predicted $\gamma / \gamma$ ' lattice mismatch of a Ni-0.6Mo-0.92Ta12.5Al-1.83Ti-10.5Cr-3.3W (at. \%) NASAIR 100 superalloy compared to an experimental determination by Nathal et al. [27]. 
Thermodynamic predictions based on the TCNI6 database provide insights into, for example, the phase stabilities for Ni-based superalloys at equilibrium states. The combination with a mobility database such as MOBNI3 makes it possible to predict the diffusivity and simulate the kinetics of some of the processes governing the evolution of the microstructure.

Ni-base superalloys have excellent strength and creep resistance, but in many applications they require protective coatings. In the NiAl-based coatings, the Al-rich $\beta$-phase (bcc-B2) serves as an $\mathrm{Al}$ reservoir for the formation of a protective $\alpha$-alumina scale. During service, the coating degrades mainly due to the interdiffusion between the coating and substrate. It is therefore important to study the interdiffusion fluxes occurring between the coating and substrate in order to optimize the lifetime of the coating. The thermodynamically compatible kinetic data in the solution phases are fundamental in such a simulation. The advantage of the present databases is that the $\gamma$ - and $\gamma^{\prime}$-phases have been modeled with a single Gibbs energy function taking into account the crystallographic relation between these two phases. Furthermore, the $\beta$-phase has been modeled with three sub-lattices (one for interstitials) which all include vacancies. This latter feature is extremely important to correctly capture the observed concentration dependency in the interdiffusion coefficients for $\beta$ NiAl.

Bypassing the limitation of the disperse diffusion model which only considers diffusion in a single continuous matrix phase, the more recently developed, so-called homogenization model, by Larsson and Engström [28] takes into account diffusion in all the stable solution phases, including the $\gamma-, \beta$ - and $\gamma^{\prime}$-phases. Using electron probe microanalysis (EPMA) Perez et al. [29] measured the concentration profiles resulting from interdiffusions in solidto-solid diffusion couples between single phase $\beta$-NiAl and various multiphase $\left(\gamma+\gamma^{\prime}\right)$ commercial Ni-base superalloys that were annealed at $1050^{\circ} \mathrm{C}$ for $96 \mathrm{~h}$. In the present paper a simulation is presented comparing with one of their experimental diffusion couples formed by joining a single phase $\beta$-NiAl with the GTD111 superalloy. Table 1 gives the initial concentration (at. $\%$ ) for the NiAl-coating and GTD111 superalloy reported in Perez et al. [29]. To simplify the calculation some elements with low concentrations reported in the experimental study are ignored in the present simulation, namely C (0.48), Mo (0.97) and Ta (0.89), while $\mathrm{Ni}$ is the balance. In addition to the initial (average) concentration profiles, we also specify the temperature $\left(1050^{\circ} \mathrm{C}\right)$ and simulation time (96h). Furthermore, $\beta-, \gamma-, \gamma^{\prime}$ - and $\sigma$-phases are considered. Thermodynamic and kinetic data are derived from TCNI6 and MOBNI3.

Table 1. Initial concentration (at\%) in respectively the NiAlcoating and GTD111 superalloy, as used in the simulation.

\begin{tabular}{|l|l|l|l|l|l|l|}
\hline & $\mathrm{Ni}$ & $\mathrm{Al}$ & $\mathrm{Co}$ & $\mathrm{Cr}$ & $\mathrm{Ti}$ & $\mathrm{W}$ \\
\hline NiAl coating & Bal. & 50.5 & & & & \\
\hline GTD111 & Bal. & 6.9 & 9.5 & 16.6 & 6.24 & 0.97 \\
\hline
\end{tabular}

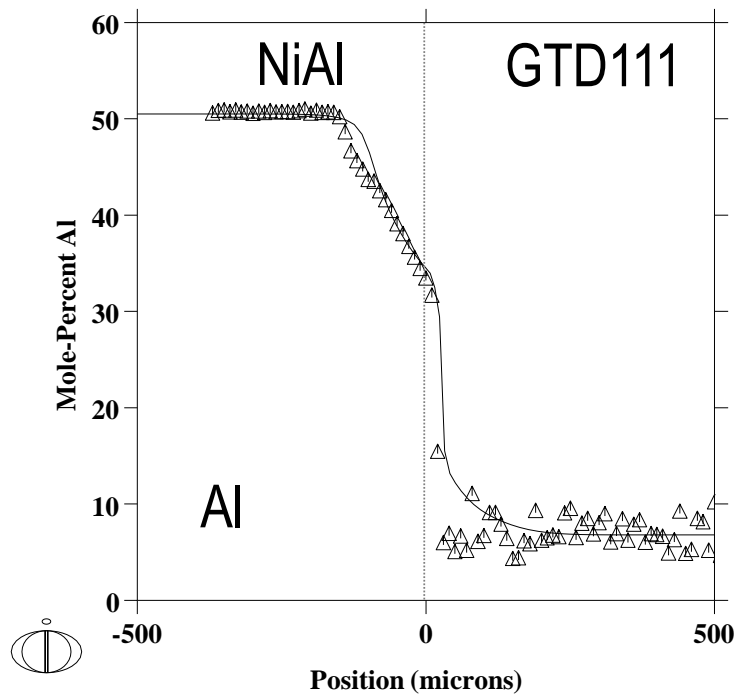

Figure 12. Comparison between calculated (curves) and experimental (symbols) information for the overall $\mathrm{Al}$ concentration profiles annealing at $1050^{\circ} \mathrm{C}$ for 96 hours in the $\beta$ $\mathrm{NiAl} / \mathrm{GTD} 111$ diffusion couple.

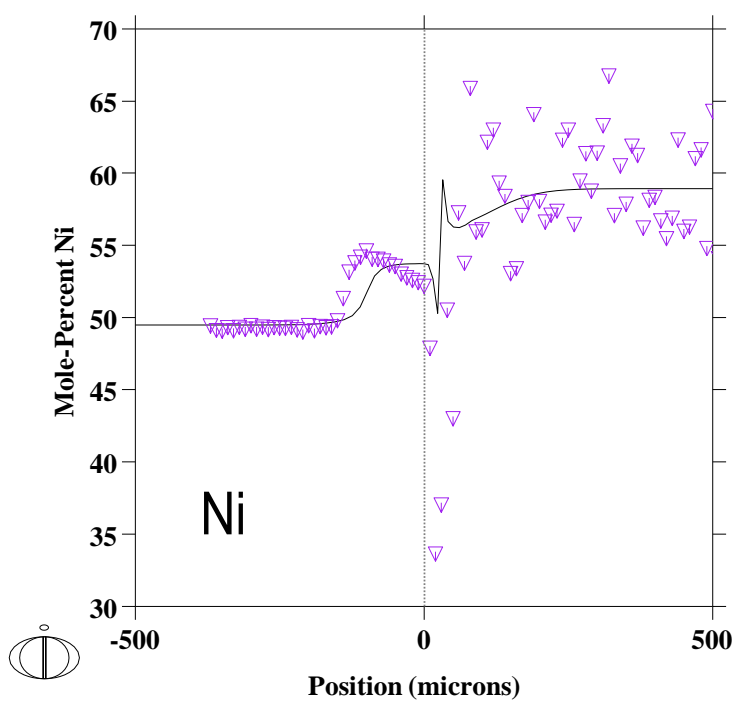

Figure 13. Comparison between calculated (curves) and experimental (symbols) information for the overall $\mathrm{Ni}$ concentration profiles after annealing at $1050^{\circ} \mathrm{C}$ for 96 hours in the $\beta$-NiAl/GTD111 diffusion couple. 


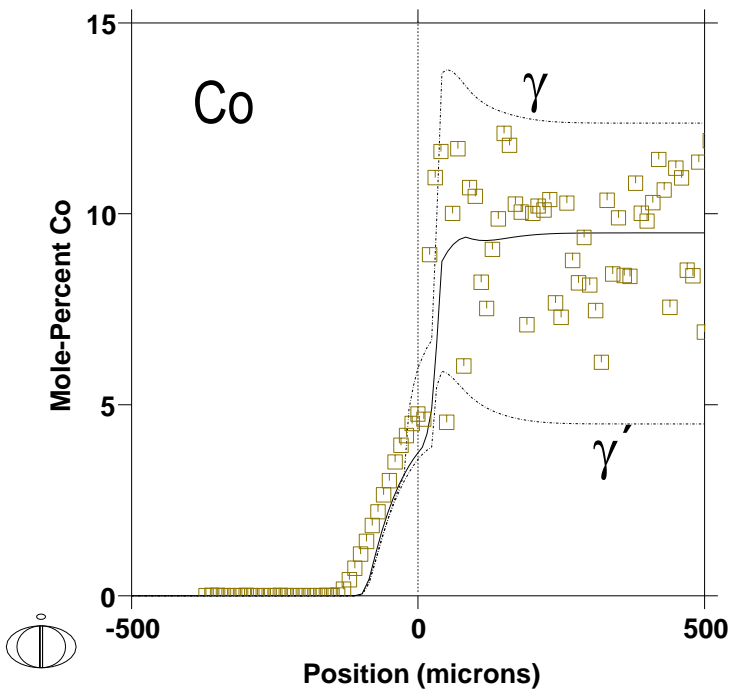

Figure 14. Concentration profile of $\mathrm{Co}$ in the $\beta-\mathrm{NiAl} / \mathrm{GTD} 111$ diffusion couple after annealing at $1050^{\circ} \mathrm{C}$ for 96 hours.

Figures 12-15 show calculated overall concentration profiles in comparison with experimental data for elements $\mathrm{Al}, \mathrm{Ni}, \mathrm{Co}, \mathrm{W}$. Symbols represent experimental data from Perez et al [29]. The dotted vertical line indicates the position of the initial interface. Despite the complex concentration profiles due to the multicomponent nature of the diffusion couple, the agreement obtained is very satisfactory as demonstrated in the figures. Both calculated and measured concentration profiles show for most elements either a peak or a trough immediately on the right to the initial interface. The experimental points in the $\gamma+\gamma^{\prime}$ two-phase region are very scattered, and this depends on if pure $\gamma$ or $\gamma^{\prime}$ phase region is hit with the electron beam or if it is a combined twophase mixture. In Figure 14 the predicted concentration profile in pure $\gamma, \gamma^{\prime}$ (dotted lines) and the overall value are shown, which explain the scattered experimental values in a satisfying way.

According to the simulation the single $\beta$-phase region extends into the GTD111 superalloy, whereas the $\gamma+\gamma^{\prime}$ two-phase region in GTD111 is receding, see Figure 16. The simulation also predicts the formation of $\sigma$-phase close to the original interface as shown in Figure 16. The formation of many small precipitates rich in refractory elements was reported in Perez et al. [29]. The peak in the experimentally determined $\mathrm{W}$ concentration profile (see Figure 15) further supports the precipitation of TCP phases such as $\sigma$-phase close to the initial interface.

By combining the thermodynamic (TCNI6) and kinetic (MOBNI3) databases available for Thermo-Calc and DICTRA with additional property data (e.g. interfacial energies), we have the necessary data input for the new precipitation modeling software, TC-PRISMA, that makes it possible to perform simulations on concurrent nucleation, growth, dissolution and coarsening in multi-component Ni-based superalloy systems.

This new computational tool is based on the Langer-Schwartz theory [30] and adopts the Kampmann-Wagner numerical approach [31] to solve the governing equation for the evolution of the particle size distribution function.

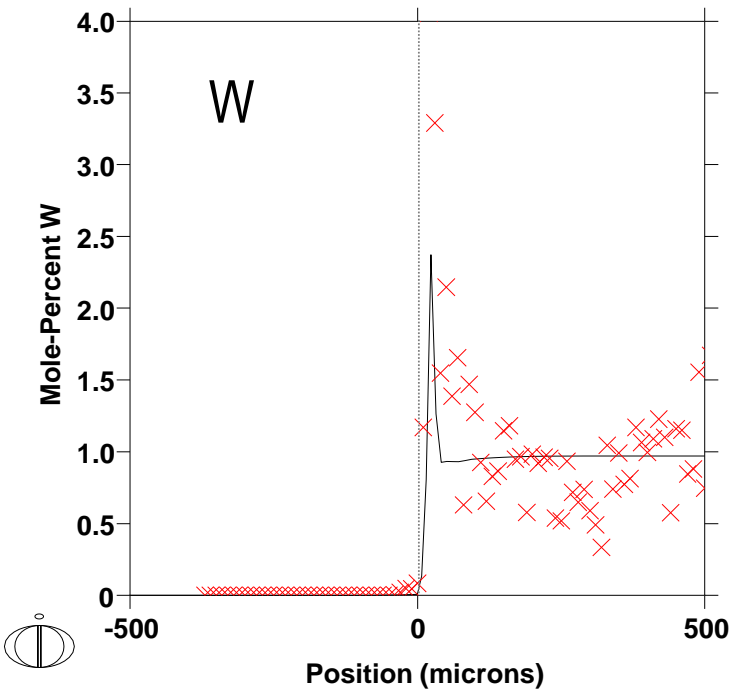

Figure 15. Comparison between calculated (curves) and experimental (symbols) information for the overall $\mathrm{W}$ concentration profiles annealing at $1050^{\circ} \mathrm{C}$ for 96 hours in the $\beta$ $\mathrm{NiAl} / \mathrm{GTD} 111$ diffusion couple.

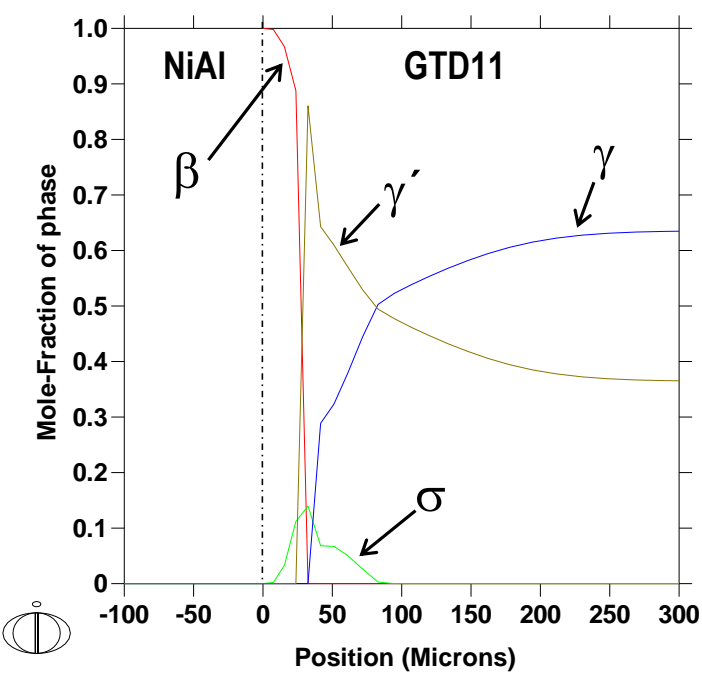

Figure 16. Calculated amount of different phases obtained from simulating annealing at $1050^{\circ} \mathrm{C}$ during 96 hours for the $\beta$ $\mathrm{NiAl} / \mathrm{GTD} 111$ diffusion couple.

A general growth rate model [32] for precipitate particles in multicomponent systems has been developed and implemented. Evolution of mean radius, number density, volume fraction, and size distribution of precipitate particles can be simulated using TC-PRISMA, see Figure 17. Nucleation rate and precipitate composition can also be obtained during the simulation. Coupled with relevant microstructure-property models, the computer program could be used to estimate the change of mechanical properties of alloys upon aging or tempering. 


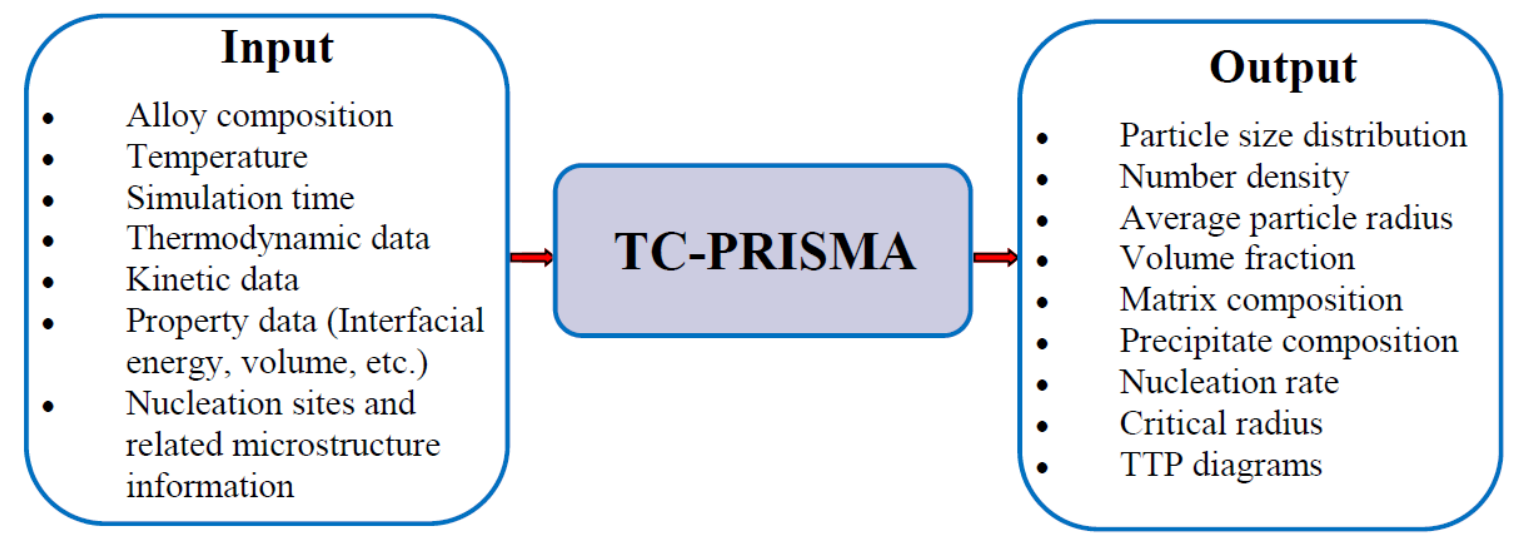

Figure 17. Overview of input and output data for TC-PRISMA.

Mao [33] measured the size evolution for $\gamma^{\prime}$ precipitates in the Rene 88 DT superalloy at composition Ni-1.99Al-12.99Co$15.67 \mathrm{Cr}-4 \mathrm{Mo}-3.88 \mathrm{~W}-3.72 \mathrm{Ti}-0.7 \mathrm{Nb}$ (mass \%) at various temperatures between 1233 and $1353 \mathrm{~K}$. Figure 18 illustrates the comparison between the predicted mean radius by TC-PRISMA, and the corresponding measurements by Mao. A reasonable agreement is obtained considering that the simulation did not involve any parameter fitting and a single value for the interfacial energy, $0.023 \mathrm{~J} / \mathrm{m}^{2}$, directly taken from Sudbrack et al. [34] has been used.

\section{Summary}

A new thermodynamic database, TCNI6, containing 25 elements (Al, Ar, B, C, Co, Cr, Fe, H, Hf, Mo, N, Nb, Ni, O, Pd, Pt, Re, Ru, $\mathrm{Si}, \mathrm{Ta}, \mathrm{Ti}, \mathrm{V}, \mathrm{W}, \mathrm{Y}, \mathrm{Zr}$ ) and 343 phases has been developed for nickel base superalloys in a true CALPHAD spirit, based on critical assessments of all possible binary systems and most Nicontaining ternary systems in full range of composition, with industry input and support, which enables predictions to be made for multicomponent systems and alloys of industrial importance. The database has also been validated where possible against higher order systems as well

The molar volume data incorporated in TCNI6 is critically assessed for most phases of importance to Ni-based Superalloys, including the $\gamma, \gamma^{\prime}, \alpha, \beta, \gamma ', \eta, \delta$, liquid, TCP-phases, carbides and borides.

All solution phases are thermodynamically modeled within the framework of the compound energy formalism (CEF). The physically reasonable modelling and critical thermodynamic evaluations give the database a good prediction of thermodynamic properties as well as the phase equilibria. For example, the ordered and disordered bcc $(\mathrm{A} 2 / \alpha$ and $\mathrm{B} 2 / \beta)$ and fcc $(\mathrm{A} 1 / \gamma$ and L12/ $\gamma^{\prime}$ ) phases are modeled with a two sub-lattice model using a single Gibbs energy curve which enables order/disorder transformations to be reproduced. The combined-CEF model for those ordered phases that do not disorder makes the correct prediction of site fraction of the sigma phase. The link between fundamental physics based models and critically assessed data also allows simulations to be performed with realistic conditions on alloys of practical importance such as the phase stability and fractions varies with temperature of a real Ni-based superalloy.
A critical requirement to successfully performing the interdiffusion simulations present in this paper is access to both accurate thermodynamic and kinetic CALPHAD-type databases. Compatible with TCNI6 a new mobility database MOBNI3 has also been developed. It contains descriptions for the atomic mobility for respectively $\gamma(\mathrm{A} 1), \gamma^{\prime}\left(\mathrm{L}_{2}\right), \alpha(\mathrm{A} 2), \beta$ (B2) and liquid phases. Combining the MOBNI3 database, in the case study of interdiffusion between NiAl coatings and Ni-base superalloy substrates, the simulation results have been validated against experimental data and the agreement is very satisfactory given the complexity of the problem.

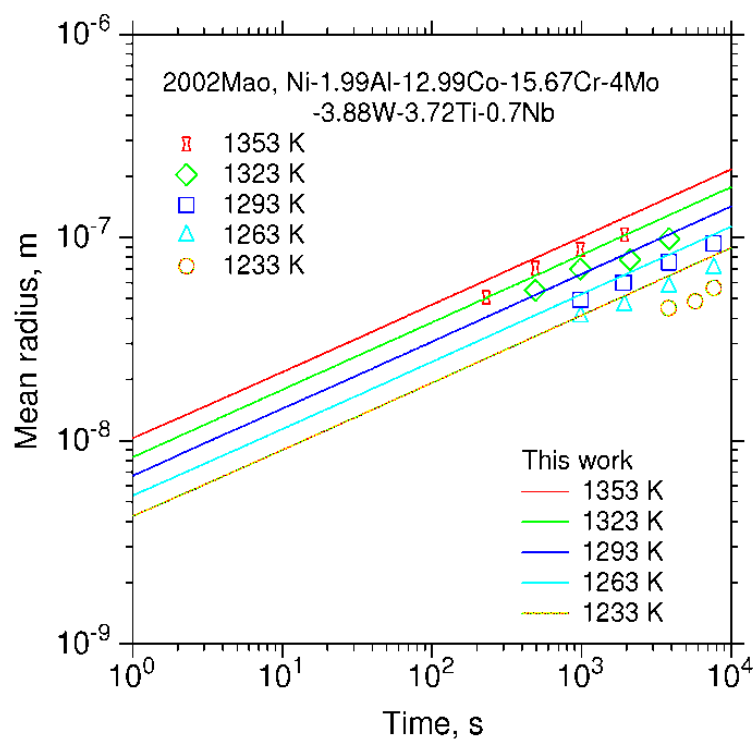

Figure 18. The predicted mean radius of the $\gamma^{\prime}$ precipitates vs holding time at different temperatures in Rene88DT by using the software TC-PRISMA and databases TCNI6+MOBNI3 compared with experimental data.

Using the new software TC-PRISMA, which simulates the kinetics of precipitation processes, some predictions have been made using these new databases combined with interface property data for the mean radius of $\gamma^{\prime}$ as a function of time and the results agree satisfactorily with the experimental data. 
Despite of the above mentioned benefits of modeling and simulation based on self-consistent thermodynamic and kinetic database, e.g. accelerating development and implementation of new materials, they do not replace the need for experiments and testing, but complement each other by feeding key data to modeling and better targeting of experiments. One may utilize real world experience, coupling with scientific understanding and prediction to reduce trial and error and minimize cost of alloy development

\section{Acknowledgments}

The authors thank Dr. Nathalie Dupin for her valuable contribution to the development of TCNI6 database and for her constructive comments on the manuscript.

\section{References}

1. L. Kauffman and H. Bernstein, Computer Calculations of Phase Diagrams with Special Reference to Refractory Metals, Academic Press, New York, NY, 1970.

2. N. Saunders and A. P. Miodownik, Calphad Calculations of Phase Diagrams: A Comprehensive guide, Pergamon Materials Series, Vol. 1, (ed. R. W. Cahn, 1998).

3. TCNI6: Thermo-Calc Ni-base alloys database, version 6.0. Developed and provided by Thermo-Calc Software AB (2012), www.thermocalc.com

4. B. Sundman, B. Jansson, and J.-O. Andersson, (1985) The Thermo-Calc databank system. Calphad, 9, 153-199.

5. J.-O. Andersson, T. Helander, L. Höglund, P.F. Shi and B. Sundman. "Thermo-Calc and DICTRA, Computational tools for materials science" Calphad, 26 (2002), 273-312.

6. MOBNI3: Thermo-calc Ni-alloys mobility database, version 2.0. Developed and provided by Thermo-Calc Software AB (2012), www.thermocalc.com

7. H. Larsson, L. Höglund, "Multiphase diffusion simulations in 1D using the DICTRA homogenization model" Calphad 33 (2009) 495 .

8. Q. Chen, G. Sterner, and H.-J. Jou, 1st world congress on integrated computational materials engineering, July 10-14 2011, Seven Springs, PA, USA.

9. H.L. Lukas, S.G. Fries, and B. Sundman, Computational Thermodynamics - The Calphad Method, (2007) Cambridge.

10. M. Hillert, J. of Alloys and Compounds, 320 (2001) 161-176.

11. M. Hillert, B. Jansson, B. Sundman and J. Ågren, Met. Mat. Trans. A, 16A (1985) 261-266.

12. B. Sundman, Calphad, 15 (1991) 109-119.

13. N. Dupin and B. Sundman, Scandinavian J. Metallurgy 30 (2001) 184-192.

14. B. Hallstedt, N. Dupin, M. Hillert, et al., Calphad, 31 (2007) 28-37.

15. X.-G. Lu, M. Selleby, B. Sundman, Calphad 29 (2005) 49-55. 16. T. Helander and J. Agren, Acta Materialia 47 (1999) 11411152 .

17. M. Hillert, L. Kjellqvist, H. Mao et al., Calphad, 33 (2009) 227-232.

18. L. Kjellqvist, M. Selleby, B. Sundman, Calphad, 32 (2008) 577-592.

19. G. Dmitrieva, V. Vasilenko, I. Melnik, Chemistry of Metals and Alloys 1 (2008) 338-342.
20. K. Shinagawa, T. Omori, J. Sato, K. Oikawa, I. Ohnuma, R. Kainuma, K. Ishia, Materials Transactions, 49 (6), 1474-1479 (2008).

21. Y. F. Cui, X. Zhang, G. L. Xu, W. J. Zhu, H. S. Liu and Z. P. Jin, J Mater Sci, 46, 2611-2621 (2011).

22. J. Joubert, Progress in Materials Science, 53 (2008) 528-583.

23. Y. Iijima, O. Taguchi, K. Hirano, Transactions of the Japan Institute of Metals (1980) 21 366-374.

24. Y. Minamino, Y. Koizumi, N. Tsuji, et al., Science and Technology of Advanced Materials (2000) $1237-249$.

25. C. Y. Cui, Y. F. Gu, D. H. Ping and H. Harada: Met. Mat. Trans. A, 40A (2009) 282-291.

26. L. Fang, Y. Wang, F. Xiao, Z. Tao, K. Mukai, Mater. Sci. Eng. B 132 (2006) 164-169.

27. M. Nathal, R. Mackay and R. Garlick: Mater. Sci. Eng. 75 (1985) 195-205.

28. H. Larsson, A. Engstrom, Acta Materialia 54 (2006) $2431-$ 2439.

29. E. Perez, T. Patterson, Y. Sohn, J Phase Eq. Diff. 27 (2006) 659-664.

30. J. Langer and A. Schwartz, Phys. Rev. A, 21 (1980) 948-958.

31. R. Wagner and R. Kampmann. "Homogeneous second phase precipitation". In: P Haasen, editor. Materials Science and Technology: A Comprehensive Treatment. Weinheim: WileyVCH, 1991. p. 213.

32. Q. Chen, J. Jeppsson, J. Agren. Acta Mater, 56 (2008) 18901896.

33. Jian Mao, Ph.D. dissertation, West Virginia University, 2002. 34. C.K. Sudbrack, R.D. Noebe, D.N. Seidman, Acta Mater, 55 (2007) 119-130. 